\title{
SYNTHESIS OF SOME POTENTIAL ANTIESTROGENIC AGENTS
}

\author{
Hany M. Safwat, Gehan H. Hegazy, Ghaneya S. Hassan \\ Department of Pharmaceutical Chemistry, Faculty of Pharmacy, Cairo University, \\ Cairo, Egypt
}

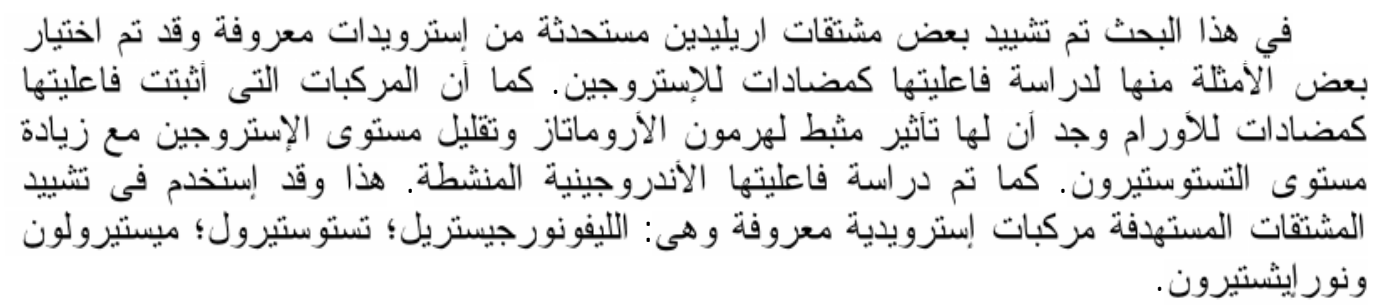

Some novel steroidal arylidene derivatives were synthesized and representative examples were tested for their antiestrogenic activity. The active compounds were subjected for antitumor activity screening. The compounds tested displayed aromatase inhibitory activity as shown by decrease in estradiol and increase in testosterone levels. Further, some compounds were tested for androgenic/anabolic activity. The steroids used for preparation of these arylidene derivatives are the clinically applied: levo-Norgestrel, Testosterone, Mesterolone and Norethisterone.

\section{INTRODUCTION}

A new series of pure estrogen antagonists is needed as a second line therapy after the failure of long term tamoxifene treatment. It was reasoned that continuous tamoxifen treatment could eventually lead to tamoxifenstimulated tumor growth. ${ }^{1}$ Toremifene is a new antiestrogen being developed for the treatment of breast cancer. ${ }^{2}$ ICI 182,780 is an estradiol derivative that was shown to reduce tumor proliferation in patients. ${ }^{3}$ In addition, ICI 182,780 may prove useful as an adjuvant agent in early stage of endometrial cancer. ${ }^{2}$ Testosterone and androgens have been known to be antiestrogenic. ${ }^{4}$ Ring $\mathrm{A}$ of most progestational agents is similar to that of testosterone. This aroused the authors'attention to utilize norgestrel as one possible synthone for attaining some of the presently described new compounds intended to act as antiestrogenies that can be used in treatment of estrogen dependent tumors.A bipartate compound was previously synthesized by the authors ${ }^{5}$ consisting of norgestrel bound to the DNA intercalator acridine-4-carboxylic acid via an ester bridge between the latter and hydrazone of the former, and was proved to possess antitumor activity in cell lines under the auspices of the NCI, Besthesda, Maryland. Further the presence of a tertiary amino group appended to a phenyloxyalkyl moiety would appear important for antiestrogenic activity as shown by tamoxifen, clomiphene, toremifene, raloxifene, idoxifene ${ }^{3}$ and mifepristone. ${ }^{6}$ Therefore the authors decided to synthesize the new compounds to be derived from the clinically known androgenic substances, testosterone, mesterolone, and the progestogenic agent, levo-norgestrel to be attached to a tertiary amino group through an alkoxyarylidene bridge $(\mathbf{1 4 - 1 7 , 2 0 , 2 1 )}$ in order to acquire the SP2 carbon atom bound to locant 2. This would abide to the carbon skeleton of antiestrogenics. Since antiestrogenic activity of tertiary amines could be lost upon oxidative demethylation, ${ }^{3}$ the authors applied the idea of substituting the dialkyl tertiary amino function with the more resistant cyclic derivatives such as morpholino and pipridino which may avoid dealkylation thus reducing metabolic degradation. 


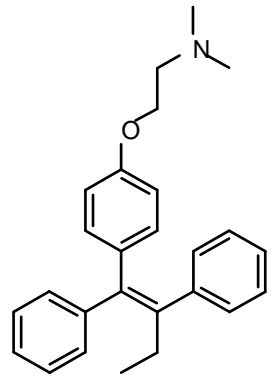

Tamoxifen<smiles>O=C(c1ccc(OCCCN2CCCCC2)cc1)c1c(-c2ccc(O)cc2)sc2cc(O)ccc12</smiles>

Raloxifene<smiles>CCN(CC)CCOc1ccc(C(=C(Cl)c2ccccc2)c2ccccc2)cc1</smiles>

Clomiphene<smiles>CC/C(=C(\c1ccccc1)c1ccc(I)cc1)c1ccc(OCCN2CCCC2)cc1</smiles>

Idoxifene<smiles>CN(C)CCOc1ccc(C(=C(CCCl)c2ccccc2)c2ccccc2)cc1</smiles>

Thamemifferme

\section{Chemistry}

Aldol condensation of the steroidal ketones, levo-norgestrel 1a, norethisterone $\mathbf{1 b}$, testosterone $\mathbf{1 c}$ and mesterolone $\mathbf{2}$ with aromatic aldehydes namely p-N,Ndimethylaminobenzaldehyde and p-N,Ndimethylaminocinnamaldehyde in alkaline medium gave the corresponding arylidene derivatives 3-9 (Scheme 1). Condensation of testosterone, levo-norgestrel and mesterolone with vanillin gave new three intermediates $\mathbf{1 0 , 1 1 , 1 8}$ respectively which reacted with 1,2dibromopropane to give compounds 12,13,19 respectively (Schemes 2 and 3). The latter three compounds reacted with morpholine and pipridine to give 14-17, 20,21.

The reaction of mesterolone $\mathbf{2}$ with aldehydes gave several spots in thin layer chromatography, the major of which was isolated by preparative TLC using pet.ether / ethyl acetate (3:7). There are two possibilities viz. 2 or 4-arylidene derivatives. Investigation of the electron densities on these two locants revealed a small difference that did not warrant preferring one possibility over the other. On the other hand, from a steric point of view the 2 and $4 \beta$-protons are under the same 1,3-impact of the C-19-angular methyl. The $1 \alpha$-methyl group assumes an axial $\alpha$-orientation quiet remote from the concerned centers (Figure 1). The literature has been in favor of the 2-locant involvement based on more favored 2-enolate formation than 4 -in the $5 \alpha$-series. ${ }^{7-9}$ For this reason resort has to be made to X-ray crystal diffraction. Unfortunately, despite the several trials using different solvents, we were unable to obtain suitable crystals for the purpose. Accordingly, based on the difference of heat of formation which was calculated using $3 \mathrm{D}$, Chem Draw version 4.0- (-124.4 for 4 and -123.4 for 2) i.e. 1 unit in favor of 4 and the possmiffee activation of the 4-position via 1,3interaction between the $1 \alpha$-methyl and the $5 \alpha$ $\mathrm{H}$, it can be assumed that the isolated product may be the 4-arylidene derivative.

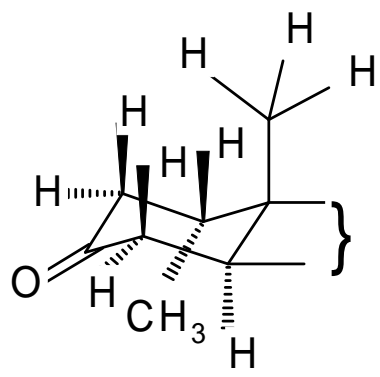

Fig. 1

\section{EXPERIMENTAL}

Melting points were determined by the open capillary method and are uncorrected using Electrothermal Digital Melting Point Apparatus 9100. Microanalyses were carried out at Microanalytical Unit, Faculty of Science, Cairo University. IR spectra $(\mathrm{KBr})$ were recorded on Schimadzu 435 Spectrophotometer. ${ }^{1} \mathrm{H}$-NMR spectra were recorded on Jeol, FX 90Q (90 MHz) using TMS as an internal standard. Mass spectra were determined on Fining SSQ 7000 Gas Chromatograph-Mass spectrometer. TLC system is pet.ether : ethyl acetate (3:7). Method of separation of mestrolone with aldehydes by preparative TLC using the same TLC system. 
The chemical nomenclature of the parent steroids, utilized in the synthesis of the target compounds, will be replaced by the term "steroidal". This shall indicate, according to Schemes 1, 2 and 3, the following: For those derived from 1a, $17 \alpha$-ethinyl-13-ethyl androst4-en-3-on-17 $\beta$-ol; from 1b, $17 \alpha$-ethinyl-19norandrost-4-en-3-on-17 $\beta$-ol; from 1c, androst4-en-3-on-17 $\beta$-ol; from $2,1 \alpha$-methylandrost-3on-17 $\beta$-ol. The term "arylidino" shall indicate, according to Scheme 1, either (4-dimethylaminobenzylidino-), or (4-dimethylaminocinnamylidino-).

Methylene hump of steroids in NMR spectra which ranges from 1.00-2.06 includes the protones of $\mathrm{CH}_{2}$ groups at $\mathrm{C} 1,6,7,11,12$, $15 \& 16$ and $\mathrm{CH}$ at $\mathrm{C} 8,9,10,14 \& 17$ if they are unsubstituted. ${ }^{10}$

\section{General method for preparing arylideno derivatives}

A mixture of steroidal ketone (0.01 mole), the corresponding aromatic aldehyde (0.01 mole) and sodium metal (0.02 mole) in $\mathrm{EtOH}$ $(20 \mathrm{ml})$ was refluxed for $2 \mathrm{~h}$. The reaction mixture was acidified with $10 \% \mathrm{HCl}$ and cooled. The precipitate was filtered, dried and crystallized from the appropriate solvent.

\section{2-Arylideno steroidal (3-7) and 4-Aryideno steroidal (8,9) (Scheme 1; Table 1).}

According to general method for preparing arylideno derivatives. Compounds 3-9 were crystallized from $\mathrm{MeOH}$.

IR $\left(\mathrm{cm}^{-1}\right)$ for 3: $3400(\mathrm{OH}), 1680(\mathrm{C}=\mathrm{O})$. IR $\left(\mathrm{cm}^{-1}\right)$ for 7: $3400(\mathrm{OH}), 1670(\mathrm{C}=\mathrm{O})$.

IR $\left(\mathrm{cm}^{-1}\right)$ for 8: $3400(\mathrm{OH}), 1710(\mathrm{C}=\mathrm{O})$. IR $\left(\mathrm{cm}^{-1}\right)$ for 9: $3400(\mathrm{OH}), 1720(\mathrm{C}=\mathrm{O})$.

${ }^{1} \mathrm{H}-\mathrm{NMR}$ of $6\left(\mathrm{CDCl}_{3}-\mathrm{D}_{2} \mathrm{O}\right) \delta \mathrm{ppm}: 0.99$ (t, $3 \mathrm{H}, \mathrm{CH}_{3}-\mathrm{CH}_{2}$ ), 1.43-1.85 (methylene hump), 2.95-3.00 (m, 8H, N( $\left(\mathrm{CH}_{3}\right)_{2}$ and $\left.\mathrm{CH}_{3}-\mathrm{CH}_{2}\right), 4.72$ (s, $1 \mathrm{H}, \mathrm{C} \equiv \mathrm{CH}), 6.80(\mathrm{~m}, 4 \mathrm{H}$, olefinic $\mathrm{H}), 7.09$ 7.18 (m, 4H, Ar H), 9.70 (s, 1H, OH exch.).

${ }^{1} \mathrm{H}-\mathrm{NMR}$ of $7\left(\mathrm{CDCl}_{3}\right) \delta \mathrm{ppm}: 0.78(\mathrm{~s}, 3 \mathrm{H}$, C18), 1.16 (s, 3H, C19), 1.20-1.53 (methylene hump), $3.06\left(\mathrm{~s}, 6 \mathrm{H}, \mathrm{N}\left(\mathrm{CH}_{3}\right)_{2}\right), 6.70(\mathrm{~m}, 4 \mathrm{H}$, olefinic H), 7.00-7.23 (m, 4H, Ar H), 9.65 (s, $1 \mathrm{H}, \mathrm{OH})$.

${ }^{1} \mathrm{H}-\mathrm{NMR}$ for $8\left(\mathrm{CDCl}_{3}\right) \delta \mathrm{ppm}: 0.80(\mathrm{~s}, 3 \mathrm{H}$, C18), 0.89 (d, 3H, C1 J= $5 \mathrm{~Hz}), 1.11$ (s, 3H, C19), 1.38-2.00 (methylene hump), 3.07 (s, 6H, $\left.\mathrm{N}\left(\mathrm{CH}_{3}\right)_{2}\right), 6.73(\mathrm{~m}, 3 \mathrm{H}$, olefinicH and $\mathrm{Ar} \mathrm{H})$, $7.67(\mathrm{~d}, 2 \mathrm{H}, \operatorname{Ar} \mathrm{H}, \mathrm{J}=5.8 \mathrm{~Hz}), 9.71(\mathrm{~s}, 1 \mathrm{H}$, $\mathrm{OH})$.

${ }^{1} \mathrm{H}-\mathrm{NMR}$ for $9\left(\mathrm{CDCl}_{3}\right) \delta \mathrm{ppm}: 0.80$ $3 \mathrm{H}, \mathrm{C} 18), 0.89$ (d, 3H, C1, J= $3 \mathrm{~Hz}$ ), 1.12 (s, 3H, C19), 1.34-1.90 (methylene hump), 3.09 (s, $\left.6 \mathrm{H}, \mathrm{N}\left(\mathrm{CH}_{3}\right)_{2}\right), 3.65(\mathrm{~m}, 3 \mathrm{H}$, olefinic $\mathrm{H}), 6.73$ (d, 2H, Ar H, J= 4 Hz), 7.76 (d, 2H, Ar H, J= 4 $\mathrm{Hz}), 9.74$ (s, 1H, OH).

$\mathrm{M}^{{ }^{+}}$for 7: Calculated 445.65 , found 444.2 $(5.57 \%)$.

$\mathrm{M}^{+}$for 8: Calculated 435.65 , found 435.20 $(0.53 \%)$.

$\mathrm{M}^{{ }^{+}}$for 9: Calculated 461.69, found $461.65(7.81 \%)$.

2- or 4-(3-methoxy-4-hydroxybenzylidinosteroidal (10,11,18) (Schemes 2 and 3; Tables 2 and 3 )

The general method for preparing arylideno derivatives was applied using vanillin as an aldehyde. The precipitate was crystallized from $\mathrm{MeOH}$.

IR $\left(\mathrm{cm}^{-1}\right)$ for 11: $3400(\mathrm{OH}), 1660(\mathrm{C}=\mathrm{O})$. IR $\left(\mathrm{cm}^{-1}\right)$ for 18: $3400(\mathrm{OH}), 1680(\mathrm{C}=\mathrm{O})$.

${ }^{1} \mathrm{H}-\mathrm{NMR}$ of $11\left(\mathrm{DMSO}_{6}, \mathrm{~d}_{6}, \mathrm{D}_{2} \mathrm{O}\right) \delta \mathrm{ppm}$ : 0.85 (broad, 3H, $\quad \underline{\mathrm{CH}}_{3}-\mathrm{CH}_{2}$ ), $1.90-1.24$ (methylene hump), 3.41 (m, 2H, $\mathrm{CH}_{3}-\mathrm{CH}_{2}$ ), $3.67\left(\mathrm{~s}, 3 \mathrm{H}, \mathrm{OCH}_{3}\right), 5.21(\mathrm{~s}, 1 \mathrm{H}, \mathrm{C} \equiv \mathrm{CH}), 6.25$ $(\mathrm{m}, 2 \mathrm{H}$, olefinic $\mathrm{H}), 6.80-7.10((\mathrm{~m}, 3 \mathrm{H}, \mathrm{Ar} \mathrm{H})$, 8.49 (s, 1H, OH exch.), 9.27 (s, 1H, Ar OH exch.).

2-or 4-[3-Methoxy-4-(2-bromo-1-propoxy)benzylideno[ steroidal $(\mathbf{1 2 , 1 3 , 1 9 )}$ (Schemes 2 and 3; Tables 2 and 3)

A mixture of arylideno derivative $\mathbf{1 0 , 1 1}$ or 18 (0.01 mole) and 1,2-dibromopropane (0.01 mole) was refluxed for $3 \mathrm{~h}$ in abs. EtOH and few drops of $\mathrm{Et}_{3} \mathrm{~N}$. Excess solvent was removed under vacuum and the product crystallized from $\mathrm{MeOH}$.

IR $\left(\mathrm{cm}^{-1}\right)$ for 19: $3500(\mathrm{OH}), 1710(\mathrm{C}=\mathrm{O})$.

2-or 4-[3-Methoxy-4-(2-N-morpholino-1propoxy) benzylideno] steroidal $(14,15,20)$ (Schemes 2 and 3; Tables 2 and 3); and 2-or-4(3-methoxy-4-(2-N-piperidino-1-propoxy) benzylideno) steroidal $(\mathbf{1 6 , 1 7 , 2 1 )}$ (Schemes 2 and 3; Tables 2 and 3)

Compounds 12,13 or 19 (0.01 mole) were refluxed with the appropriate secondary amines (morpholine or piperidine) $(0.01 \mathrm{~mole})$ in 
Table 1: Physical properties and microanalytical data of the new compounds (3-9).

\begin{tabular}{|c|c|c|c|c|c|c|c|c|c|}
\hline No & $\mathrm{R}^{1}$ & $\mathrm{R}^{2}$ & $\mathrm{R}^{3}$ & $\mathrm{Ar}$ & $\begin{array}{l}\text { Mol. Formula } \\
\text { (M.wt) }\end{array}$ & M.P, ${ }^{\circ}$ & $\begin{array}{c}\text { Yield } \\
\%\end{array}$ & \multicolumn{2}{|c|}{$\begin{array}{l}\text { Microanalysis \% } \\
\text { Calculated Found }\end{array}$} \\
\hline 3 & $\mathrm{H}$ & $\mathrm{C}_{2} \mathrm{H}_{5}$ & $\mathrm{C} \equiv \mathrm{CH}$ & $\overline{\mathrm{C}_{6} \mathrm{H}_{4} \mathrm{~N}\left(\mathrm{CH}_{3}\right)_{2}}$ & $\begin{array}{c}\mathrm{C}_{30} \mathrm{H}_{37} \mathrm{NO}_{2} \\
(443.63)\end{array}$ & $\begin{array}{c}240 \\
- \\
241 \\
\end{array}$ & 60 & $\begin{array}{l}\text { C } 81.22 \\
\text { H } 8.41 \\
\text { N } 3.16 \\
\end{array}$ & $\begin{array}{l}80.90 \\
8.70 \\
3.42 \\
\end{array}$ \\
\hline 4 & $\mathrm{H}$ & $\mathrm{CH}_{3}$ & $\mathrm{C} \equiv \mathrm{CH}$ & $\mathrm{C}_{6} \mathrm{H}_{4} \mathrm{~N}\left(\mathrm{CH}_{3}\right)_{2}$ & $\begin{array}{c}\mathrm{C}_{29} \mathrm{H}_{35} \mathrm{NO}_{2} \\
(429.60)\end{array}$ & $\begin{array}{c}122 \\
- \\
125\end{array}$ & 40 & $\begin{array}{l}\text { C } 81.08 \\
\text { H } 8.21 \\
\text { N } 3.26\end{array}$ & $\begin{array}{l}80.90 \\
8.10 \\
3.22\end{array}$ \\
\hline 5 & $\mathrm{CH}_{3}$ & $\mathrm{CH}_{3}$ & $\mathrm{H}$ & $\mathrm{C}_{6} \mathrm{H}_{4} \mathrm{~N}\left(\mathrm{CH}_{3}\right)_{2}$ & $\begin{array}{c}\mathrm{C}_{28} \mathrm{H}_{37} \mathrm{NO}_{2} \\
(419.61)\end{array}$ & $\begin{array}{c}118 \\
- \\
120\end{array}$ & 50 & $\begin{array}{l}\text { C } 80.15 \\
\text { H } 8.89 \\
\text { N } 3.34\end{array}$ & $\begin{array}{l}80.00 \\
9.20 \\
3.15\end{array}$ \\
\hline 6 & $\mathrm{H}$ & $\mathrm{C}_{2} \mathrm{H}_{5}$ & $\mathrm{C} \equiv \mathrm{CH}$ & $\begin{array}{c}\mathrm{CH}=\mathrm{CH}- \\
\mathrm{C}_{6} \mathrm{H}_{4} \mathrm{~N}\left(\mathrm{CH}_{3}\right)_{2}\end{array}$ & $\begin{array}{c}\mathrm{C}_{32} \mathrm{H}_{39} \mathrm{NO}_{2} \\
(469.67)\end{array}$ & $\begin{array}{c}190 \\
- \\
193 \\
\end{array}$ & 45 & $\begin{array}{l}\text { C } 81.83 \\
\text { H } 8.37 \\
\text { N } 2.98 \\
\end{array}$ & $\begin{array}{l}81.60 \\
8.30 \\
2.50 \\
\end{array}$ \\
\hline 7 & $\mathrm{CH}_{3}$ & $\mathrm{CH}_{3}$ & $\mathrm{H}$ & $\begin{array}{c}\mathrm{CH}=\mathrm{CH}- \\
\mathrm{C}_{6} \mathrm{H}_{4} \mathrm{~N}\left(\mathrm{CH}_{3}\right)_{2}\end{array}$ & $\begin{array}{c}\mathrm{C}_{30} \mathrm{H}_{39} \mathrm{NO}_{2} \\
(445.65)\end{array}$ & $\begin{array}{c}210 \\
- \\
212\end{array}$ & 65 & $\begin{array}{l}\text { C } 80.85 \\
\text { H } 8.82 \\
\text { N } 3.14\end{array}$ & $\begin{array}{l}80.90 \\
8.70 \\
2.83\end{array}$ \\
\hline 8 & - & - & - & $\mathrm{C}_{6} \mathrm{H}_{4} \mathrm{~N}\left(\mathrm{CH}_{3}\right)_{2}$ & $\begin{array}{c}\mathrm{C}_{29} \mathrm{H}_{41} \mathrm{NO}_{2} \\
(435.65)\end{array}$ & $\begin{array}{c}163 \\
- \\
165\end{array}$ & 90 & $\begin{array}{l}\text { C } 79.95 \\
\text { H } 9.48 \\
\text { N } 3.22\end{array}$ & $\begin{array}{l}80.10 \\
9.50 \\
3.20\end{array}$ \\
\hline 9 & - & - & - & $\begin{array}{c}\mathrm{CH}=\mathrm{CH}- \\
\mathrm{C}_{6} \mathrm{H}_{4} \mathrm{~N}\left(\mathrm{CH}_{3}\right)_{2}\end{array}$ & $\begin{array}{c}\mathrm{C}_{31} \mathrm{H}_{43} \mathrm{NO}_{2} \\
(461.69)\end{array}$ & $\begin{array}{c}240 \\
- \\
242\end{array}$ & 60 & $\begin{array}{l}\text { C } 80.65 \\
\text { H } 9.39 \\
\text { N } 3.03\end{array}$ & $\begin{array}{l}80.45 \\
9.10 \\
2.72\end{array}$ \\
\hline
\end{tabular}

Table 2: Physical properties and microanalytical data of the new compounds (10-17).

\begin{tabular}{|c|c|c|c|c|c|c|c|c|}
\hline No & $\mathrm{R}^{1}$ & $\mathrm{R}^{2}$ & $\mathrm{R}^{3}$ & $\begin{array}{l}\text { Mol. Formula } \\
\text { (M.wt) }\end{array}$ & M.P, ${ }^{\circ}$ & Yield \% & \multicolumn{2}{|c|}{$\begin{array}{l}\text { Microanalysis \% } \\
\text { Calculated Found }\end{array}$} \\
\hline 10 & $\mathrm{CH}_{3}$ & $\mathrm{CH}_{3}$ & $\mathrm{H}$ & $\begin{array}{c}\mathrm{C}_{27} \mathrm{H}_{34} \mathrm{O}_{4} \\
(422.57)\end{array}$ & $132-135$ & 55 & $\begin{array}{l}\text { C } 76.74 \\
\text { H } 8.11\end{array}$ & $\begin{array}{l}76.70 \\
7.50\end{array}$ \\
\hline 11 & $\mathrm{H}$ & $\mathrm{C}_{2} \mathrm{H}_{5}$ & $\mathrm{C} \equiv \mathrm{CH}$ & $\begin{array}{l}\mathrm{C}_{29} \mathrm{H}_{34} \mathrm{O}_{4} \\
(446.59)\end{array}$ & $114-115$ & 95 & $\begin{array}{l}\text { C } 77.99 \\
\text { H } 7.67\end{array}$ & $\begin{array}{l}78.22 \\
7.30\end{array}$ \\
\hline 12 & $\mathrm{CH}_{3}$ & $\mathrm{CH}_{3}$ & $\mathrm{H}$ & $\begin{array}{c}\mathrm{C}_{30} \mathrm{H}_{39} \mathrm{BrO}_{4} \\
(543.63) \\
\end{array}$ & $100-102$ & 40 & $\begin{array}{l}\text { C 66.28 } \\
\text { H 7.23 } \\
\end{array}$ & $\begin{array}{l}67.16 \\
7.00 \\
\end{array}$ \\
\hline 13 & $\mathrm{H}$ & $\mathrm{C}_{2} \mathrm{H}_{5}$ & $\mathrm{C} \equiv \mathrm{CH}$ & $\begin{array}{c}\mathrm{C}_{32} \mathrm{H}_{39} \mathrm{BrO}_{4} \\
(567.66)\end{array}$ & $120-121$ & 30 & $\begin{array}{l}\text { C } 67.71 \\
\text { H } 6.92\end{array}$ & $\begin{array}{l}67.98 \\
6.72\end{array}$ \\
\hline 14 & $\mathrm{CH}_{3}$ & $\mathrm{CH}_{3}$ & $\mathrm{H}$ & $\begin{array}{c}\mathrm{C}_{34} \mathrm{H}_{47} \mathrm{NO}_{5} \\
(549.75)\end{array}$ & $168-169$ & 75 & $\begin{array}{l}\text { C } 74.28 \\
\text { H } 8.62 \\
\text { N } 2.54\end{array}$ & $\begin{array}{l}74.24 \\
8.01 \\
2.40\end{array}$ \\
\hline 15 & $\mathrm{H}$ & $\mathrm{C}_{2} \mathrm{H}_{5}$ & $\mathrm{C} \equiv \mathrm{CH}$ & $\begin{array}{c}\mathrm{C}_{36} \mathrm{H}_{47} \mathrm{NO}_{5} \\
(573.78)\end{array}$ & $242-245$ & 45 & $\begin{array}{l}\text { C 75.36 } \\
\text { H } 8.26 \\
\text { N } 2.44 \\
\end{array}$ & $\begin{array}{l}75.60 \\
7.90 \\
2.46 \\
\end{array}$ \\
\hline 16 & $\mathrm{CH}_{3}$ & $\mathrm{CH}_{3}$ & $\mathrm{H}$ & $\begin{array}{c}\mathrm{C}_{35} \mathrm{H}_{49} \mathrm{NO}_{4} \\
(547.78)\end{array}$ & $200-203$ & 50 & $\begin{array}{l}\text { C } 76.74 \\
\text { H } 9.02 \\
\text { N } 2.56 \\
\end{array}$ & $\begin{array}{l}76.57 \\
9.36 \\
2.12 \\
\end{array}$ \\
\hline 17 & $\mathrm{H}$ & $\mathrm{C}_{2} \mathrm{H}_{5}$ & $\mathrm{C} \equiv \mathrm{CH}$ & $\begin{array}{c}\mathrm{C}_{37} \mathrm{H}_{49} \mathrm{NO}_{4} \\
(571.81)\end{array}$ & $157-160$ & 40 & $\begin{array}{l}\text { C } 77.72 \\
\text { H } 8.64 \\
\text { N } 2.45\end{array}$ & $\begin{array}{l}78.01 \\
8.90 \\
2.39\end{array}$ \\
\hline
\end{tabular}


Table 3: Physical properties and microanalytical data of the new compounds (18-21).

\begin{tabular}{|c|c|c|c|c|c|}
\hline No. & $\begin{array}{l}\text { Mol. Formula } \\
\text { (M.W) }\end{array}$ & M.P., ${ }^{\circ}$ & Yield \% & \multicolumn{2}{|c|}{$\begin{array}{l}\text { Microanalysis \% } \\
\text { Calculated Found }\end{array}$} \\
\hline 18 & $\begin{array}{c}\mathrm{C}_{28} \mathrm{H}_{38} \mathrm{O}_{4} \\
(438.61) \\
\end{array}$ & $280-283$ & 65 & $\begin{array}{l}\text { C } 76.68 \\
\text { H } 8.73 \\
\end{array}$ & $\begin{array}{l}76.91 \\
8.95\end{array}$ \\
\hline 19 & $\begin{array}{c}\mathrm{C}_{31} \mathrm{H}_{43} \mathrm{BrO}_{4} \\
(559.68) \\
\end{array}$ & $>300$ & 50 & $\begin{array}{l}\text { C } 66.53 \\
\text { H } 7.74 \\
\end{array}$ & $\begin{array}{l}66.26 \\
7.51 \\
\end{array}$ \\
\hline 20 & $\begin{array}{c}\mathrm{C}_{35} \mathrm{H}_{51} \mathrm{NO}_{5} \\
(565.79)\end{array}$ & $218-220$ & 20 & $\begin{array}{l}\text { C } 74.30 \\
\text { H } 9.08 \\
\text { N } 2.48 \\
\end{array}$ & $\begin{array}{l}74.01 \\
8.90 \\
2.36 \\
\end{array}$ \\
\hline 21 & $\begin{array}{c}\mathrm{C}_{36} \mathrm{H}_{53} \mathrm{NO}_{4} \\
(563.82)\end{array}$ & $196-198$ & 20 & $\begin{array}{l}\text { C } 76.69 \\
\text { H } 9.47 \\
\text { N } 2.48 \\
\end{array}$ & $\begin{array}{l}76.41 \\
9.72 \\
2.40 \\
\end{array}$ \\
\hline
\end{tabular}

\section{Scheme 1}

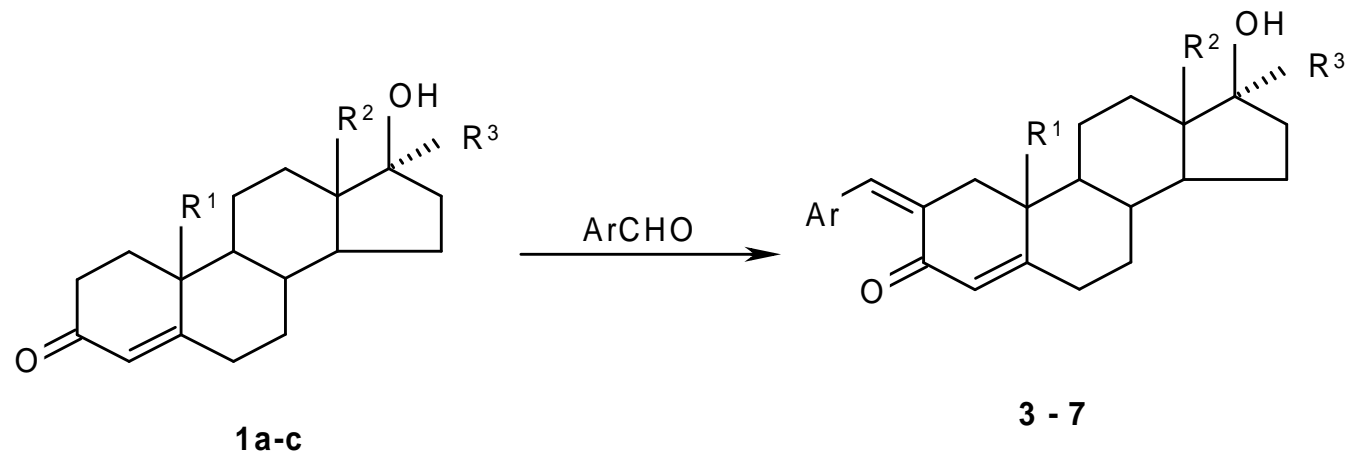

1a $\mathrm{R}^{1}: \mathrm{H}, \mathrm{R}^{2}: \mathrm{C}_{2} \mathrm{H}_{5}, \mathrm{R}^{3}: \mathrm{C} \equiv \mathrm{CH}$

1b $\mathrm{R}^{1}: \mathrm{H}, \mathrm{R}^{2}: \mathrm{CH}_{3}, \mathrm{R}^{3}: \mathrm{C} \equiv \mathrm{CH}$

1c $R^{1}: \mathrm{CH}_{3}, \mathrm{R}^{2}: \mathrm{CH}_{3}, \mathrm{R}^{3}: \mathrm{H}$<smiles>C[C@H]1CC(=O)CC2CCC3C4CCC(O)C4(C)CCC3C21C</smiles>

2

\section{$\mathrm{ArCHO}$}<smiles>C[C@H]1CC(=O)/C(=C\[Al])[C@H]2CCC3C4CCC(O)C4(C)CCC3C12C</smiles>

8,9

Ar:<smiles>CC=Cc1ccc(N(C)C)cc1</smiles> 


\section{Scheme 2}

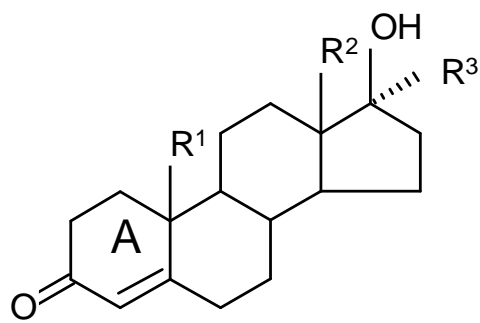<smiles>CCC(=O)C1CCC(=Cc2ccc(O)c(OC)c2)C(=O)C1</smiles>

$1 a, 1 c$

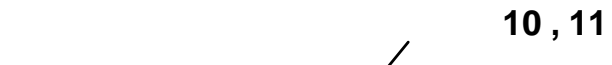<smiles>COc1cc(/C=C2\CC(=O)C(C)(C)CC2=O)ccc1OCC(C)C</smiles><smiles>C1CCNCC1</smiles><smiles>COc1ccc(C=C2C(=O)CC(C)C(C)C2=O)cc1OC</smiles>

14,15<smiles>COc1cc(C=C2CCC(C(=O)CC(C)(C)C)CC2)ccc1OCC(C)C</smiles> 


\section{Scheme 3}

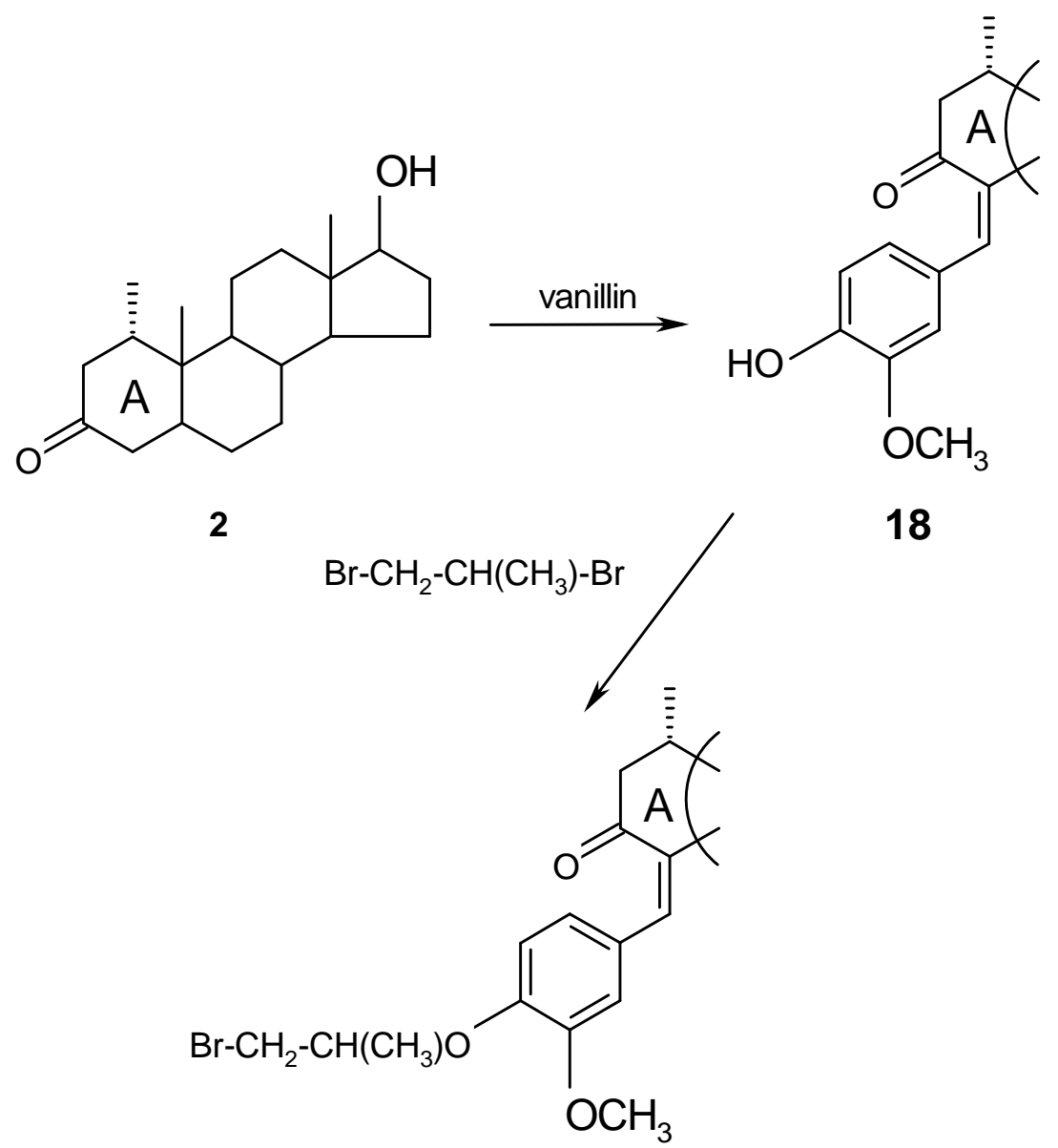

19
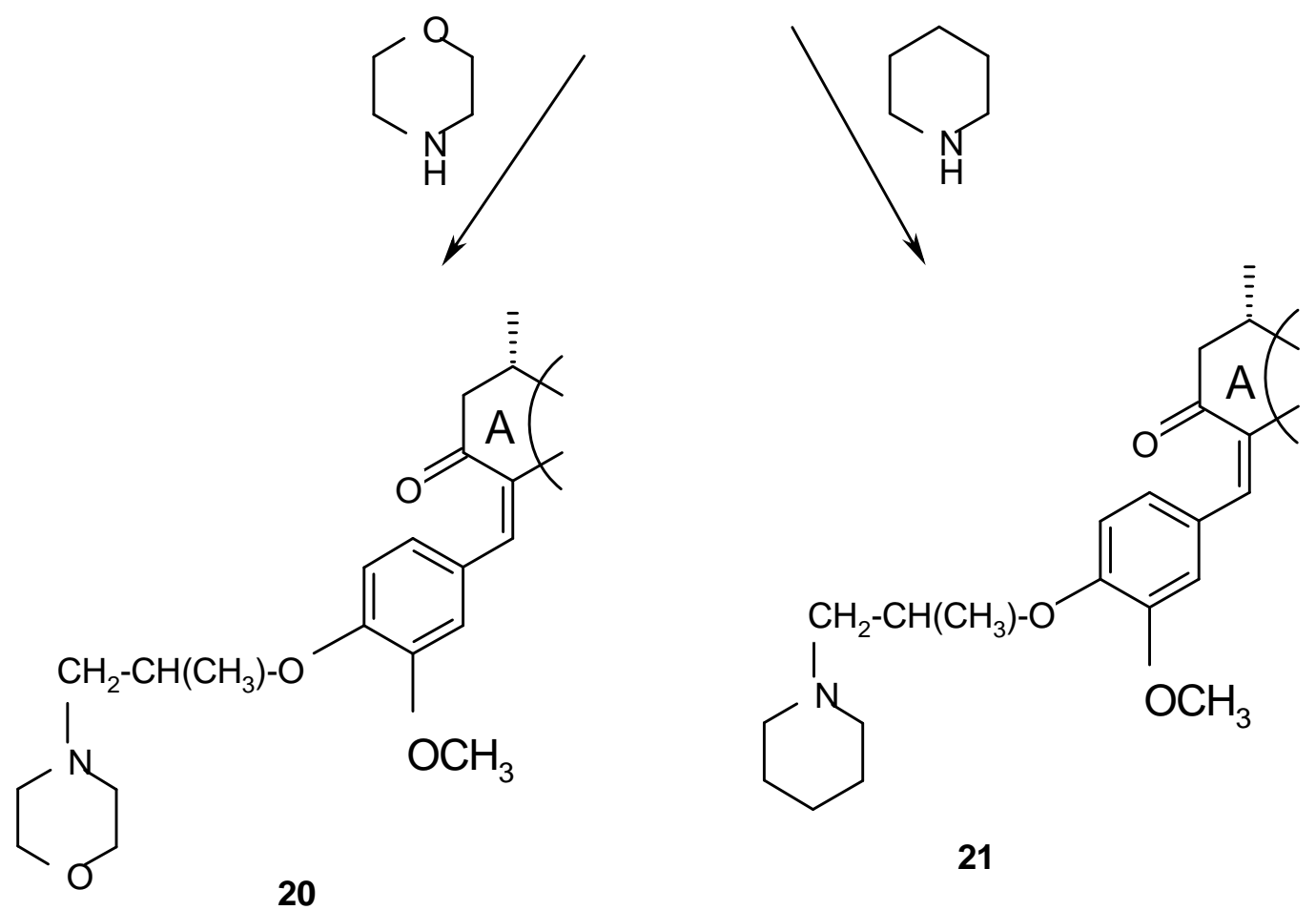
abs.EtOH (20 ml) for $12 \mathrm{~h}$. Excess solvent was removed under vacuum and reaction mixture was then poured on ice $\mathrm{H}_{2} \mathrm{O}$. The precipitate was filtered, dried and crystallized from EtOH / $\mathrm{H}_{2} \mathrm{O}$.

IR $\left(\mathrm{cm}^{-1}\right)$ for 14: $3450(\mathrm{OH}), 1680(\mathrm{C}=\mathrm{O})$.

${ }^{1} \mathrm{H}-\mathrm{NMR}$ for $14\left(\mathrm{CDCl}_{3}\right) \delta \mathrm{ppm}: 0.81(\mathrm{~s}$, $3 \mathrm{H}, \quad \mathrm{C} 18), \quad 0.97 \quad(\mathrm{~s}, \quad 3 \mathrm{H}, \quad \mathrm{C} 19), \quad 1.00-1.60$ (methylene hump and $\mathrm{CH}_{2}-\mathrm{CH}\left(\mathrm{CH}_{3}\right) \mathrm{O}$ ), 1.87$2.20\left(\mathrm{~m}, 4 \mathrm{H},\left(\mathrm{CH}_{2}\right)_{2} \mathrm{~N}\right), 3.00\left(\mathrm{~d}, 2 \mathrm{H}, \mathrm{CH}_{2}-\right.$ $\left.\mathrm{CH}\left(\mathrm{CH}_{3}\right) \mathrm{O}\right), 3.70\left(\mathrm{~m}, 4 \mathrm{H},\left(\mathrm{CH}_{2}\right)_{2} \mathrm{O}\right), 3.90(\mathrm{~m}$, $\left.1 \mathrm{H}, \mathrm{CH}_{2}-\mathrm{CH}\left(\mathrm{CH}_{3}\right) \mathrm{O}\right), 3.97\left(\mathrm{~s}, 3 \mathrm{H}, \mathrm{OCH}_{3}\right)$, $6.96(\mathrm{~s}, 2 \mathrm{H}$, olefinic $\mathrm{H}), 7.40(\mathrm{~m}, 3 \mathrm{H}, \mathrm{Ar} \mathrm{H})$, $9.80(\mathrm{~s}, 1 \mathrm{H}, \mathrm{OH})$.

${ }^{1} \mathrm{H}-\mathrm{NMR}$ for $15\left(\mathrm{CDCl}_{3}\right) \delta \mathrm{ppm}: 0.99(\mathrm{t}$, $3 \mathrm{H}, \underline{\mathrm{CH}_{3}}-\mathrm{CH}_{2}$ ), 1.25-1.6 (methylene hump and $\mathrm{CH}_{2}-\mathrm{CH}\left(\mathrm{CH}_{3}\right) \mathrm{O}$ ), 2.17-2.31 (broad, $4 \mathrm{H}$, $\left.\left(\mathrm{CH}_{2}\right)_{2} \mathrm{~N}\right), 2.90\left(\mathrm{~d}, 2 \mathrm{H}, \mathrm{CH}_{2}-\mathrm{CH}\left(\mathrm{CH}_{3}\right) \mathrm{O}\right), 3.41$ (q, $\left.2 \mathrm{H}, \mathrm{CH}_{3}-\mathrm{CH}_{2}\right), 3.52-3.56\left(\mathrm{~m}, 4 \mathrm{H},\left(\mathrm{CH}_{2}\right)_{2} \mathrm{O}\right)$, 3.68-3.78 (m, $\left.1 \mathrm{H}, \mathrm{CH}_{2}-\mathrm{CH}\left(\mathrm{CH}_{3}\right) \mathrm{O}\right), 3.83$ (s, $\left.3 \mathrm{H}, \mathrm{OCH}_{3}\right), 5.53(\mathrm{~s}, 1 \mathrm{H}, \mathrm{C} \equiv \mathrm{CH}), 7.04(\mathrm{~m}, 2 \mathrm{H}$, olefinic H), 7.26-7.51 (m, 3H, Ar H),11.91 (s, $1 \mathrm{H}, \mathrm{OHexch})$.

${ }^{1} \mathrm{H}-\mathrm{NMR}$ for $20\left(\mathrm{CDCl}_{3}\right) \delta \mathrm{ppm}: 0.83(\mathrm{~s}$, $3 \mathrm{H}, \mathrm{C} 18), 0.88$ (d, 3H, C1, J=6.9 Hz), 1.12 (s, $3 \mathrm{H}, \mathrm{C} 19$ ), 1.25-1.90 (methylene hump and $\left.\mathrm{CH}_{2}-\mathrm{CH}\left(\mathrm{CH}_{3}\right) \mathrm{O}\right), 2.10-2.30\left(\mathrm{~m}, 4 \mathrm{H},\left(\mathrm{CH}_{2}\right)_{2} \mathrm{~N}\right)$, $2.70\left(\mathrm{~d}, 2 \mathrm{H}, \mathrm{CH}_{2}-\mathrm{CH}\left(\mathrm{CH}_{3}\right) \mathrm{O}\right), 3.27(\mathrm{~m}, 4 \mathrm{H}$, $\left.\left(\mathrm{CH}_{2}\right)_{2} \mathrm{O}\right), 4.00\left(\mathrm{~s}, 3 \mathrm{H}, \mathrm{OCH}_{3}\right), 4.14(\mathrm{~m}, 1 \mathrm{H}$, $\left.\mathrm{CH}_{2}-\mathrm{CH}\left(\mathrm{CH}_{3}\right) \mathrm{O}\right), 6.9(\mathrm{~s}, 1 \mathrm{H}$, olefinic $\mathrm{H}), 7.25$ (m, 3H, Ar H), 9.50 (s, 1H, OH).

$\mathrm{M}^{+}$for 20: Calculated 565.79, found $565.80(24.81 \%)$.

\section{Biological screening}

Compounds 3,6-9,14,16 and 21 were tested in vitro for cytotoxic activity. Aromatase inhibitory activity as well as anabolic/ androgenic ratio were evaluated for compounds 3, 9, 14 and 16.

\section{I- Cytotoxic activity ${ }^{11}$}

Cultures fixed with trichloroacetic acid were stained for $30 \mathrm{~min}$. with $0.4 \%$ (w/v) sulforhodamine B dissolved in $1 \%$ acetic acid. Unbound dye was removed by four washes with $1 \%$ acetic acid and protein-bound dye was extracted for determination of optical density. Compounds were tested against 3 cell lines namely brain tumor cell line (U251), mammary carcinoma cell line (MCF7) and cervix carcinoma cell line (Hela).

\section{II- Aromatase inhibitory activity ${ }^{12}$}

Ovarian tissues from adult golden hamster were used where the ovaries were incubated in presence and in absence of the test compounds 3,9,14 and 16; using 4-hydroxy androstendione as standard at concentration $(0.03-0.3 \mathrm{mg})$. At the end of the experiment the ovaries were removed where estrogen, progesterone and testosterone were determined by radioimmunoassay. Compounds 3,9,14 and 16 were assayed and the results are shown in Table 4 using testosterone as a standard.

Table 4: Aromatase inhibitory activity.

\begin{tabular}{||c|c|c||}
\hline Compd. & $\begin{array}{c}\text { \% Reduction } \\
\text { in estrogen }\end{array}$ & $\begin{array}{c}\text { \% Increase in } \\
\text { testosterone }\end{array}$ \\
\hline $\mathbf{3}$ & 75 & 50 \\
\hline $\mathbf{9}$ & 66 & 30 \\
\hline $\mathbf{1 4}$ & 80 & 53 \\
\hline $\mathbf{1 6}$ & 75 & 50 \\
\hline
\end{tabular}

\section{III- valuation of anabolic / androgenic ratio}

They were studied by the method of Hershburger et al. ${ }^{13}$ The ratio of the weight gain of the levator ani-muscle and the weight gain of the ventral prostate gland was calculated. The gain in the weight of levator ani-muscle indicated the anabolic effect and the gain in the weight of ventral prostate gland indicated the androgenic effect. Results are shown in Table 5.

Table 5: Anabolic / androgenic ratio.

\begin{tabular}{|c|c|c|}
\hline Compd. & $\begin{array}{c}\text { Weight gain } \\
\text { prostate gland } \\
(\mathrm{mg})\end{array}$ & $\begin{array}{c}\text { Weight gain in } \\
\text { levator muscle } \\
(\mathrm{mg})\end{array}$ \\
\hline $\mathbf{3}$ & 0.15 & 2.51 \\
\hline $\mathbf{9}$ & 0.2 & 1.07 \\
\hline $\mathbf{1 4}$ & 0.19 & 2.38 \\
\hline $\mathbf{1 6}$ & 0.21 & 1.01 \\
\hline
\end{tabular}

\section{RESULTS AND CONCLUSION}

All the tested compounds were found inactive in the cytotoxic activity test against three cell lines. Compounds 3, 9, 14 and 16 showed significant aromatase inhibitory activity. The most potent compound as anti- 
estrogen was $\mathbf{1 4}$, which is a derivative of male hormone testosterone. The same compound $\mathbf{1 4}$ showed more anabolic activity than androgenic

\section{REFERENCES}

1- M.D.Wolf, S. M. L. Fahey, C. J. Parker, R. M. Cague and V. C. Jordon, J. Natl. Cancer Inst., 85, 806 (1993).

2- R. M. O. Regon, A. Cisneros, G. M. England, J. I. M. Gregor, H. D. Muenzner, V. J. Assikis, M. M. Bilimoria, M. Piete, Y. P. Dragan, H. C. Pitot, R. Chaherton and V. C. Jordan, J. Natl. Cancer Inst., 90, 1552 (1998).

3- P. L. Munson, R. A. Muller and G. R. Breese, "Principles of Pharmacology", Basic Concepts \& Clinical Application. U.S.A: Chapman and Hall, 1995, p. 828.

4- L. M. Tierney, S. T. McPhee and M. A. Papadakis, "Current Medical Diagnosis and Treatment", England: Appleton and Lang, $38^{\text {th }}$ ed., 1999, p. 81.
5- H. Safwat, K. El-Said, S. Rashad, and G. Hegazy, Bull. Pharm. Cairo Univ., 37, 21 (1999).

6- N. Engl, J. Med., 329, 804-12 (1993).

7- E. Jcory and R. A. Sneen, J. American Chem. Society, 77, 2505 (1955).

8- R. Jacquesy and J. Lvisalles, Bull. Soc. Chim France, 1942 (1967).

9- A. B. Turner, and H. I. Ringold, J. Chem. Soc., 1720 (1967).

10- M. S. Bhacca and D. H. Williams, "Application of NMR Spectroscopy in Organic Chemistry Illustaration for Steroid Field", Holden Day. $9^{\text {th }}$ edn, (1964).

11- P. Skehan, R. Storeng, D. Scudiero, A. Monks, J. Mcmahon, D. Vistica and M. Boyd, J. Natl. Cancer Inst., 1107 (1990).

12- A. Hausler, L. Schenkel, C. Krahenbuhl, G. Monnet, and A. Bhatnagar, J. Steroid Bioch., 33, 125 (1989).

13- L. G. Hershburger, G. Shebley and R. K. Meyer, Proc. Soc. Biol. Med., 83, 175 (1953). 